\title{
Dieta e câncer gástrico: aspectos históricos associados ao padrão de consumo alimentar no estado do Pará
}

\author{
Diet and gastric cancer: historical aspects associated \\ with dietary patterns in the state of Pará, Brazil
}

Ana Lúcia da Silva RESENDE²

Inês Echenique MATTOS 3

Sergio KOIFMAN ${ }^{3}$

RE S U M O

O câncer gástrico ainda constitui importante problema de saúde pública no estado do Pará, onde as taxas de mortalidade apresentam valores acima da média brasileira. As bases históricas de ocupação de espaço na Amazônia, bem como as relações econômico-sociais estabelecidas ao longo desse processo, marcaram o padrão alimentar desse Estado. Este trabalho tem por objetivo reconstruir as principais características do padrão alimentar dessa população no século passado. Procurou-se identificar todas as publicações científicas disponíveis que relacionavam dados sobre os hábitos alimentares dos residentes do Pará no século XX. A partir dessas publicações, foi possível caracterizar o padrão alimentar da população paraense no século XX, que incluía um elevado consumo de sal, utilizado na conservação de carnes e frutos do mar, um consumo relativamente reduzido e irregular de legumes e verduras e uma importante ingestão de glicídios, a partir da farinha de mandioca, muitas vezes adicionada de corantes artificiais. O padrão de dieta retratado, provavelmente comum a outras regiões da Amazônia, pode ser caracterizado, com base na literatura científica, como potencialmente favorecedor de certas condições associadas à carcinogênese gástrica. Nesse sentido, ele poderia ter contribuído, em parte, para a alta mortalidade por essa neoplasia registrada na região Norte.

Termos de indexação: dieta; farinha de mandioca; neoplasias gástricas; sal.

A B S T R A C T

Gastric cancer still constitutes an important public health problem in the state of Pará, Brazil, where mortality rates are above the Brazilian average rates. The historical basis of occupation of the Amazon region, as well as

\footnotetext{
1 Artigo elaborado a partir da dissertação de A.L.S. RESENDE, intitulada "Mortalidade por câncer gástrico e hábito alimentar no estado do Pará, 1980 - 1997”. Escola Nacional de Saúde Pública/Fundação Oswaldo Cruz; 2002.

2 Secretaria Executiva de Estado de Saúde Pública do Pará. Belém, PA, Brasil.

3 Departamento de Epidemiologia e Métodos Quantitativos em Saúde, Escola Nacional de Saúde Pública, Fundação Oswaldo Cruz. Rua Leopoldo Bulhões, 1480, Manguinhos, Rio de Janeiro, RJ, Brasil. Correspondência para/Correspondence to: I.E. MATTOS.E-mail:<imattos@ensp.fiocruz.br>.
} 
the social-economic relations established during this process, have marked the dietary patterns of this state. The aim of this work is to reconstruct some of the main characteristics of the dietary pattern of the population of Pará in the last century. We sought to identify all the scientific papers that contained data on the dietary habits of the population of Pará during the XX Century. This material allowed us to characterize the eating pattern of this population during the XX Century which included an elevated consumption of salt, used to preserve meats and seafood, a relatively low and irregular consumption of legumes and leaves and an important consumption of carbohydrates with the cassava flour being the main source and frequently containing dyestuffs. This dietary pattern, probably common to other regions of the Amazon, can be characterized as potentially supportive of certain conditions associated with gastric carcinogenesis. In this sense it could have contributed, at least partially, to the high mortality rates from this neoplasia in the Amazon region of Brazil.

Indexing terms: diet; manihot flour; stomach neoplasms; salt.

\section{N T R O D U Ç Ã O}

O câncer gástrico vem apresentando uma diminuição da incidência e da mortalidade em vários países, inclusive no Brasil, embora ainda seja uma das localizações tumorais mais freqüentes no mundo. Howson et al. ${ }^{1}$ sugeriram que o declínio das taxas de mortalidade por câncer de estômago, observado em vários países do mundo, se relacionou a modificações na dieta das diferentes populações, que adotaram padrões de consumo relacionados a um menor risco para essa neoplasia.

Em estudo que examinou o padrão de mortalidade por câncer de estômago, no período 1980-1997, no Estado do Pará e em sua capital, foi observada uma tendência decrescente constante da mortalidade por essa neoplasia², embora as taxas de mortalidade, em Belém, no período 1995-97, fossem ainda superiores às verificadas no período 1980-1985, em outras capitais de Estados brasileiros. No Estado do Pará, o câncer de estômago foi a primeira causa de morte por neoplasia em 1999 e 2000, correspondendo, respectivamente, a $15,98 \%$ e $13,99 \%$ das mortes ${ }^{3}$.

Os principais fatores da dieta implicados, segundo a literatura, na carcinogênese gástrica são a ingestão de altas concentrações de nitratos/nitritos, presentes em carnes salgadas ou em conserva, o consumo de alimentos que favorecem a formação de nitrosaminas e a ingestão excessiva de sal e amido e de alimentos mal conservados ${ }^{4,5}$.

Os tumores de estômago evoluem a partir de um processo de transformação da mucosa gástrica, que se desenvolve a longo prazo, e é originado pela ação de diferentes fatores de risco. Embora a causalidade do câncer gástrico ainda não esteja bem estabelecida, numerosos estudos epidemiológicos têm demonstrado que a dieta é o fator de risco exógeno mais relevante ${ }^{6}$. Segundo Hwang e colaboradores ${ }^{7}$, a dieta parece estar envolvida nos estágios mais precoces da transformação das células normais em células cancerosas (Figura 1). Os estágios iniciais de gastrite crônica e atrofia parecem ser promovidos pela ingestão excessiva de sal, estando os estágios intermediários associados à ingestão de nitratos, nitritos e outros fatores que favorecem a produção intragástrica de nitrosaminas. $\mathrm{O}$ alto consumo de amido também estaria envolvido nesse processo, ocasionando irritação da mucosa gástrica ou promovendo a nitrosação dos nutrientes ingeridos ${ }^{8}$. Por outro lado, agentes anti-oxidantes, como o ácido ascórbico e a vitamina $\mathrm{E}$, presentes em frutas e vegetais, poderiam inibir a produção dessas substâncias, atuando como fatores de proteção contra a carcinogênese gástrica9 .

Nesse sentido, é interessante resgatar o padrão histórico de dieta da população paraense, no século passado, que poderia ter contribuído, parcialmente, para a mortalidade por câncer gástrico registrada no Estado.

\section{MÉ T O D O S}

Para a caracterização do padrão alimentar da população paraense, tendo em vista suas possíveis repercussões no padrão de distribuição 


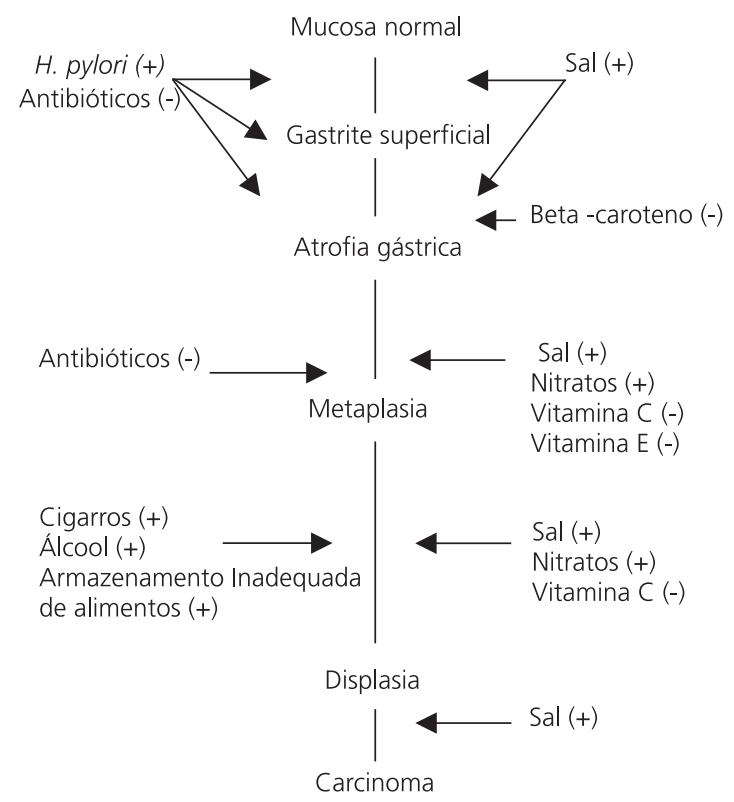

Figura 1. Influências ambientais no processo de carcinogênese gástrica*.

Fonte: *Adaptado de Hwang et al. ${ }^{30}$

da mortalidade por câncer gástrico no estado, interessava o consumo no século passado, em especial, na primeira metade daquele e entre 1950-1980. Dados mais atuais de consumo alimentar (década de 90, em diante), devido à necessidade de um período de latência entre a exposição e o desenvolvimento de neoplasia, iriam repercutir no padrão de adoecimento de anos mais recentes. Assim, no período de janeiro a dezembro de 2000, procurou-se identificar as publicações que relacionavam dados sobre hábitos alimentares da população residente no Estado do Pará, durante o século XX, para a descrição desse perfil e do contexto histórico que Ihe deu origem.

Com essa finalidade, foram conduzidas buscas no acervo de bibliotecas, museus, universidades e órgãos públicos localizados no Estado do Pará, para levantamento das referências bibliográficas disponíveis sobre o consumo alimentar da população durante o século XX. Foram consultados os bancos de dados informatizados Medline e Lilacs, com a utilização das palavras-chave "dieta" "Pará" "hábitos alimentares" "consumo alimentar". Buscas adicionais nas listas de referências das publicações identificadas e consultas a especialistas foram, também, efetuadas.

A partir do conjunto de publicações identificado, procurou-se levantar os hábitos alimentares da população paraense no século passado, em dois diferentes momentos, e caracterizar o padrão de consumo alimentar regional predominante e suas possíveis relações com a carcinogênese gástrica.

\section{RESULTADOS}

\section{Consumo alimentar no Estado do Pará}

Na primeira metade do século $X X$, a população do Estado do Pará, que vivia dispersa no interior da floresta ou à margem dos rios, tinha como principais fontes de alimentação a fauna e a flora. Segundo relatos da literatura, a base alimentar tinha origem no extrativismo, incluindo o consumo de frutas, caça e peixes em abundância, e na agricultura de subsistência, a partir de pequenas lavouras de mandioca, com a qual se preparava farinha, fonte de carboidratos na dieta das populações na Amazônia ${ }^{10}$.

Em relação aos habitantes das cidades e regiões com densidade populacional relativamente mais elevada, constituídos, em sua maioria, por assalariados ou agricultores com baixo poder aquisitivo, a ingestão protéica na dieta alimentar, tradicionalmente, era à base de peixe seco salgado, carne salgada (charque) ou em conser$v^{10}{ }^{10}$. O cálculo do consumo de proteína animal, efetuado com base no abate do gado nos matadouros, poderia induzir a falsas conclusões sobre essa ingestão, uma vez que a carne bovina in natura era rara ou inexistente no interior do Estado. Naquele período, a população urbana experimentava o efeito periódico da escassez de alimentos, pois a maioria dos produtos alimentícios precisava ser comprada ${ }^{10}$.

Os fatos acima apontados dificultam que se tenha, atualmente, uma avaliação precisa do consumo médio de alimentos per capita na primeira metade do século passado. Contudo, 
considerando a Região Amazônica no seu conjunto, e salvo raras exceções, a diversidade regional do padrão alimentar era mais importante do que a sua uniformidade, sendo aquela determinada por uma série de fatores que levavam à variação dos hábitos de alimentação em função do ambiente ${ }^{11}$.

Nesse período, o consumo de peixe, no litoral, e nas margens dos rios, era predominante no padrão alimentar, enquanto no interior prevalecia o consumo de carne de caça, e na região das ilhas do delta do Amazonas, proliferavam as plantações de açaí, fruto de palmeira regional, fonte de cálcio e carboidratos ${ }^{10}$.

Embora o solo do Estado do Pará fosse considerado de baixa fertilidade, suas terras eram, preferencialmente, agricultáveis. Em 1965, segundo o Anuário Estatístico do Brasil do Instituto Brasileiro de Geografia e Estatística12, de sua superfície de $1248042 \mathrm{~km}^{2}$, somente $2242 \mathrm{~km}^{2}$ $(0,18 \%$ da área do Estado) foram cultivados com cultura de subsistência alimentar.

Os principais alimentos de origem vegetal produzidos pelo Estado nos anos 60 eram arroz, milho, feijão, mandioca e castanha da Amazônia; quanto aos alimentos de origem animal, destacavam-se a carne bovina, leite, peixes e mariscos, ovos e aves ${ }^{13}$. Entre os produtos de origem vegetal, os mais consumidos encontram-se especificados na Tabela 1, na qual se pode observar o importante aumento na produção de mandioca (119\%) e arroz (110\%), entre 1956-65.

A produção elevada de mandioca era destinada, principalmente, ao preparo de farinha, por meio de processo artesanal de beneficiamento. Em 1976, a produção de farinha atingiu 1004322 toneladas, um acréscimo de $21 \%$, considerando como referência o ano de $1970^{14}$. A farinha de mandioca representava um dos principais componentes da dieta alimentar da população da região Norte, cuja média de consumo era de $176 \mathrm{~g} /$ pessoa/dia, naquele período ${ }^{14}$.

O consumo de hortaliças e legumes, tradicionalmente reduzido em função de características culturais, veio decrescendo ao longo do tempo, em grande parte, devido ao fato de que a produção local era insignificante e esses alimentos, quase todos importados, apareciam no mercado em pequena quantidade e com preço elevado ${ }^{14}$. O consumo médio de hortaliças e legumes era da ordem de $30 \mathrm{~g} /$ dia per capita, considerado muito reduzido, observando-se um consumo per capita de apenas 2g/dia de folhosos; entre os legumes, o maior consumo verificado era o de abóbora, que alcançava $17 \mathrm{~g} / \mathrm{dia}$, em média, e o consumo de frutas era um dos mais elevados da região Amazônica, em torno de 162g/dia, devido, principalmente, ao açaí ${ }^{13}$.

A escassez de dados sobre o consumo de frutas, e até mesmo sobre sua produção em períodos anteriores, permitiu apenas a apresentação das informações que constam na Tabela 2 , referentes ao período 1975-76. Os dados disponíveis referem-se, entretanto, a apenas dez espécies, não tendo sido encontrados registros referentes à produção de algumas frutas regionais, como açaí, pupunha, bacuri e cupuaçu, que têm um alto consumo pela população paraense.

Tabela 1. Distribuição do volume de produção dos produtos da dieta de maior consumo. Pará. 1956-1965.

\begin{tabular}{|c|c|c|c|c|c|c|}
\hline \multirow{2}{*}{ Cultura } & \multicolumn{2}{|c|}{ Produção (t) } & \multirow{2}{*}{ Acréscimo (\%) } & \multicolumn{2}{|c|}{ kg/ano Per capita } & \multirow{2}{*}{ Acréscimo (\%) } \\
\hline & 1956 & 1965 & & 1956 & 1965 & \\
\hline Mandioca & 440,042 & 964,514 & 119,000 & 69,000 & 107,000 & 55,000 \\
\hline Arroz & 32,313 & 67,955 & 110,000 & 21,000 & 38,000 & 81,000 \\
\hline Milho & 22,091 & 37,043 & 68,000 & 17,000 & 21,000 & 24,000 \\
\hline Feijão & 5,323 & 7,276 & 37,000 & 4,400 & 4,000 & $-10,000$ \\
\hline
\end{tabular}

Fonte: Instituto Brasileiro de Geografia e Estatística ${ }^{12}$. 
Tabela 2. Produção de frutas (t) no Estado do Pará, 1974-76.

\begin{tabular}{|c|c|c|c|c|c|c|c|c|}
\hline \multirow{2}{*}{ Especificação } & \multicolumn{4}{|c|}{ Produção em toneladas } & \multirow{2}{*}{$\%(*)$} & \multicolumn{3}{|c|}{ Índice } \\
\hline & 1974 & 1975 & 1976 & Total & & 1974 & 1975 & 1976 \\
\hline Banana & 5644 & 8583 & 9446 & 23673 & 57,6 & 100 & 152 & 167 \\
\hline Abacaxi & 1797 & 1070 & 445 & 3362 & 8,2 & 100 & 60 & 30 \\
\hline Coco seco & 994 & 1103 & 1026 & 3123 & 7,6 & 100 & 111 & 103 \\
\hline Manga & 1197 & 528 & 930 & 2655 & 8,5 & 100 & 44 & 77 \\
\hline Mamão & 631 & 919 & 953 & 2503 & 6,1 & 100 & 146 & 151 \\
\hline Melancia & 664 & 615 & 1122 & 2401 & 5,8 & 100 & 93 & 169 \\
\hline Maracujá & 368 & 552 & 1250 & 2170 & 5,3 & 100 & 150 & 340 \\
\hline Laranja & 240 & 339 & 294 & 873 & 2,1 & 100 & 141 & 122 \\
\hline Abacate & 63 & 109 & 53 & 225 & 0,6 & 100 & 173 & 84 \\
\hline Limão & $\cdots$ & 53 & 19 & 72 & 0,2 & - & 100 & 36 \\
\hline Total & 11598 & 13871 & 15588 & 41057 & 100,0 & 100 & 119 & 134 \\
\hline
\end{tabular}

*Percentual sobre a produção total de frutas no período 1975-76.

Fonte: Instituto Brasileiro de Geografia e Estatística ${ }^{15}$.

Nota: No cálculo dos índices foi tomado 1974 para ano base, exceção feita para o limão (1975).

O consumo per capita de proteínas de origem animal, no Estado do Pará, apresentava um índice elevado na segunda metade do século XX. Grande parte da carne era proveniente de outros estados. No matadouro de Belém, o maior centro consumidor do Estado do Pará, cerca de $30 \%$ do gado abatido na década de 60 era procedente do Estado de Goiás, sendo que cerca de 3441 toneladas de carne (2 500 toneladas ou $80 \%$, na forma de charque) eram importadas anualmente ${ }^{13}$. Devido à necessidade de importação, havia um acesso restrito a esse produto ${ }^{13} \mathrm{e}$ o alto consumo de proteínas de origem animal pode ser atribuído ao suprimento de outras fontes desse nutriente, como leite, ovos $^{10}$ e peixe, consumido com grande freqüência pela população ${ }^{13}$. Deve-se destacar, por exemplo, que a proporção de geladeiras disponíveis em Belém, entre as famílias com renda situada no percentil 40 dessa distribuição, era de apenas 14\%, naquele período ${ }^{15}$.

No final dos anos 60, a pecuária despontou como nova riqueza, revelando-se uma alternativa para a ocupação dos vazios demográficos. Nessa mesma época, a pesca continental e marítima se encontrava em pleno desenvolvimento, assumindo especial dimensão, devido à extensão e à variedade dos recursos hídricos ${ }^{16}$. Naquele período, coexistiam, no setor pesqueiro estadual, uma atividade essencialmente artesanal, que respondia pela maior parcela da produção de pescado, e um setor empresarial, que se organizou sob o estímulo dos incentivos fiscais ${ }^{11}$.

Quanto ao consumo de carne de caça, Bastos \& Ferreira ${ }^{17}$ listaram as espécies de animais silvestres preferidas como alimento no Estado do Pará, destacando a paca, o veado, o porco do mato, a tartaruga, a anta e a cutia. Na verdade, a carne de caça se destacava, principalmente, como estratégia de sobrevivência de colonos e caboclos, ao longo da rodovia Transamazônica, construída na década de 70. Segundo Moran ${ }^{18}$, antes da construção da rodovia, os animais de caça eram muito mais comuns, mas a destruição da floresta para a agricultura afastou-os para áreas distantes, ainda não penetradas por grupamentos humanos.

Ayres ${ }^{19}$ menciona que a chegada daquela rodovia, com a conseqüente integração que originou, trouxe modificações na composição da dieta dos habitantes da região, destacando o importante papel dos fatores econômicos e culturais nessas alterações. 


\section{Características da dieta regional do Pará relacionados ao processo de carcinogênese gástrica na literatura científica}

Aqui se buscou demarcar características específicas do padrão alimentar regional do paraense do século passado, no que diz respeito a aspectos que, com base nas evidências da literatura científica, poderiam estar relacionados à etiologia do câncer de estômago. Dessa forma, são retomadas algumas questões relativas ao padrão observado no período analisado.

Embora o exame do consumo médio anual per capita dos itens da alimentação, sem considerar a estratificação social das populações, não seja a abordagem mais adequada, comparativamente ao emprego de dados epidemiológicos, provenientes de inquéritos nutricionais, a ausência de informações sobre estes últimos conduz à adoção de outros indicadores. A partir das informações disponíveis na literatura, é possível reconstruir algumas das principais características do padrão alimentar dessa população, que poderiam estar associadas à carcinogênese gástrica, especialmente a partir dos anos 60, quando já estavam disponíveis informações mais detalhadas sobre o consumo

Uma dessas características consiste no elevado consumo da farinha de mandioca, presente no cotidiano das refeições regionais. Essa farinha, produzida com o beneficiamento do tubérculo, freqüentemente é acrescida de diferentes corantes à base de anilina, o que the confere tonalidades diversas. Sendo esse um item importante na alimentação das populações amazônicas, de todas as faixas etárias, - o consumo médio anual per capita em Belém, em 1995/96, foi da ordem de $33,9 \mathrm{~kg}$, sendo de $18,0 \mathrm{~kg}$ em Salvador, 0,6kg em Goiânia e 1,2kg em Porto Alegre $^{20}$ - sua ingestão diária pode implicar em exposição química contínua.

Em relação aos hábitos alimentares regionais do passado recente, vale a pena mencionar, também, o consumo diário de tacacá, uma refeição servida com um caldo fervente, acompanhado de goma de mandioca, tucupi e camarões salgados, ingerido em temperatura elevada. Outro importante item da dieta regional, derivado da mandioca, é o tucupi, molho ácido que integra diversos pratos da cozinha paraense e de outras áreas da Amazônia (consumo per capita anual de 0,35kg em Belém) ${ }^{21}$.

O consumo de carne vermelha e peixe, dadas as dificuldades de conservação de alimentos perecíveis, na ausência de refrigeradores, era garantido graças à sua preservação, mediante a adição de sal. Dessa forma, o charque e os frutos do mar salgados, sobretudo peixe e camarão, foram incorporados ao padrão alimentar do Pará e de outras áreas da região amazônica, constituindo itens relevantes na alimentação. Comparativamente com outras regiões do país, o consumo médio populacional de peixe e camarão salgado, na década de 80 , era de 1,05kg/ano em Belém, ou seja, 10 a 15 vezes superior ao observado em Salvador $(0,12 \mathrm{~kg} / \mathrm{ano})$ e em Goiânia $(0,07 \mathrm{~kg} / \mathrm{ano})^{21}$. Em relação à ingestão de carnes preservadas, o consumo per capita em Belém, no mesmo período, foi de $2,9 \mathrm{~kg} / \mathrm{ano}$, sendo de $0,5 \mathrm{~kg} / \mathrm{ano}$ em Porto Alegre e de 0,1 kg/ano em Goiânia ${ }^{21}$. Deve-se, ainda, mencionar o consumo de carnes curadas (presunto, mortadela, salsicha, lingüiça, toucinho), da ordem de 1,1 kg/ano em Belém, na década de $90^{20}$.

O consumo regional de frutas poderia ser considerado como regular e o de legumes, como reduzido. O consumo médio per capita de banana, em Belém, foi da ordem de $8,1 \mathrm{~kg}$ na década de 90 versus $1,7 \mathrm{~kg}$ em Porto Alegre e 2,0kg em Goiânia; para laranja, foi, respectivamente, de $8,3 \mathrm{~kg}, 7,9 \mathrm{~kg}$ e 5,7kg; para outras frutas, como manga, tangerina, maracujá e maçã, foi observado um consumo médio per capita de, respectivamente, $5,2 \mathrm{~kg}, 12,9 \mathrm{~kg}$ e $8,2 \mathrm{~kg}^{20}$. Deve-se acrescentar a essas estimativas, o consumo da polpa da fruta açaí, da ordem de $11,9 \mathrm{~kg}$ per capita/ano em Belém, e de consumo ínfimo nas demais capitais, na década de 90, bem como o de outras frutas regionais, como o cupuaçu, o bacuri e a acerola. 
Em relação à ingestão de hortaliças, foi observado um consumo estável em Belém, entre as décadas de 80 e 90 (2,1 kg/ per capita/ano), mas bem menor do que o observado em Goiânia $(4,7 \mathrm{~kg})$ e Porto Alegre $(3,1 \mathrm{~kg})$. O consumo populacional médio de legumes selecionados, ao longo das mesmas décadas, foi, igualmente, mais reduzido em Belém (1,5kg/ano), versus 2,9kg/ano em Porto Alegre e 6,0kg/ano em Goiânia ${ }^{20}$.

\section{I S C U S S Ã O}

Considerando o padrão alimentar apresentado, pode-se concluir que a alimentação básica da população paraense, no século passado, incluía um consumo elevado de sal, utilizado na conservação de carnes e frutos do mar, um consumo relativamente reduzido e irregular de legumes e verduras e uma importante ingestão de glicídios, a partir da farinha de mandioca, muitas vezes adicionada de corantes artificiais com anilina. Esse padrão de dieta, provavelmente, presente em outras regiões da Amazônia, pode ser caracterizado como potencialmente favorecedor de certas condições associadas ao processo de desenvolvimento do câncer gástrico.

Ainda que não seja possível a confirmação a partir de dados empíricos referentes ao estado do Pará, é razoável supor, com base na literatura, que as precárias condições de saneamento ambiental daquele período, acrescidas da existência de aglomerações intradomiciliares, poderiam ter favorecido a distribuição da infecção por Helicobacter pylori na população desde a primeira infância, relacionada à maior prevalência de câncer gástrico ${ }^{7,22}$. Diferentes estudos mostram prevalências elevadas de $H$. pylori em populações de países em desenvolvimento, populações de baixo nível sócioeconômico, assim como em grupos etários mais jovens ${ }^{23-26}$. O papel da $H$. pylori nesse processo ainda não está claramente definido, mas o estimulo à multiplicação celular e a atração de polimorfonucleares representa hipóteses em investigação ${ }^{22}$. Assim, as agressões contínuas à mucosa gástrica, decorrentes da ação irritativa do consumo de sal, e/ou da ingestão de alimentos em temperatura elevada, poderiam atuar como facilitadores no processo de sua invasão por Helicobacter pylori e patógenos relacionados, tal como sugerido na literatura ${ }^{27}$.

Da mesma forma, o consumo de anilina, presente em vários tipos de farinha de mandioca e fonte de radicais $\mathrm{NH}_{2}$ e $\mathrm{NO}_{2}$, poderia atuar como substrato para a formação endógena de nitrosaminas, importantes carcinógenos associados à patogênese do câncer gástrico, particularmente na situação de carência relativa de anti-oxidantes ${ }^{9}$ (em função de um consumo reduzido de legumes e vegetais, embora maior de frutas). Além disso, o consumo elevado de carboidratos, característica da dieta típica das populações amazônicas em geral, poderia, também, atuar como um fator de risco para o desenvolvimento dessa neoplasia, embora seu papel no processo de carcinogênese gástrica seja, ainda, motivo de controvérsias ${ }^{3,4,8}$.

O intervalo de tempo decorrido entre as exposições associadas à dieta regional, aqui delineadas, e a observação atual de elevados níveis de câncer gástrico no Pará, asseguraria um período de latência razoavelmente extenso, para supor sua possível contribuição para a distribuição epidemiológica dessa doença. Assim, se poderia supor que vários componentes da dieta, historicamente presentes nessas populações (alto consumo de sal e carboidratos, ingestão contínua de anilina, reduzido consumo de legumes e verduras, entre outros), conjuntamente com a infecção potencialmente precoce pelo H. pylori, gerariam condições compatíveis, segundo o conhecimento científico, com o desencadeamento do câncer gástrico.

Estudos epidemiológicos publicados na última década têm propiciado um maior conhecimento do papel da dieta na morbimortalidade por doenças não transmissíveis em geral, e por neoplasias em particular. As evidências da literatura apontam para a nutrição como um dos principais determinantes modificáveis dessas doenças, demonstrando que alterações da dieta têm fortes efeitos, tanto positivos como negativos, 
sobre a saúde humana ${ }^{28}$. Essas mudanças podem influenciar, não somente a condição de saúde atual dos indivíduos, mas determinar o desenvolvimento, ou não, de uma doença não transmissível, ao longo da vida ${ }^{29}$.

Como fator importante na promoção e manutenção da saúde, durante todo o curso da vida, a nutrição ocupa posição proeminente em atividades de prevenção ${ }^{29}$. É preciso entender melhor as relações entre o consumo alimentar e a ocorrência de neoplasias, para que se possa propor medidas preventivas. Para isso, é importante dispor de informações sobre padrões de dieta, atuais e pregressos de portadores de câncer.

Nesse sentido, a caracterização do padrão alimentar do Pará, no século XX, pretende contribuir para ampliar a compreensão sobre a primazia assumida pelo câncer de estômago, como uma das localizações tumorais de maior incidência naquele Estado, e em outros grupos populacionais da região Amazônica.

\section{REFERÊ N CIAS}

1. Howson CP, Hiyama T, Wynder EL. The decline in gastric cancer: epidemiology of an unplanned triumph. Epidemiol Rev. 1986; 8:1-27.

2. Resende ALS. Mortalidade por câncer gástrico e hábito alimentar no Estado do Pará - 1980 - 1997. Dissertação de Mestrado. Escola Nacional de Saúde Pública. Fundação Oswaldo Cruz; 2002.

3. Brasil. Ministério da Saúde. Datasus Sistema de Informações sobre Mortalidade [acesso 11 set. 2000]. Disponível em: http://www.datasus. gov.br

4. Kono S, Hirohata T. Nutrition and stomach cancer Cancer Causes and Control. 1996; 7(1):41-55.

5. La Vecchia C, Levi F, Lucchini F, Nigri E, Boyle P. Trends in cancer mortality in the USSR, 1965-1990. Int J Cancer. 1994; 56(1):31-9.

6. Nomura AM, Hankin J, Kolonel LN, Wilkins LR, Goodman MT, Stemmermann GN. Case-control study of diet and other risk factors for gastric cancer in Hawai (United Status). Cancer Causes Control. 2003; 14(6):547-58.

7. Grew KD, Neugut Al. Epidemiology of gastric cancer. World J Gastroenterol. 2006; 12(3): 354-62.

8. Mayne ST, Risch HÁ, Dubrow R, Chow W, Gammon $M D$, Vaughan TL, et al. Nutrient intake and risk of subtypes of esophageal and gastric cancer. Cancer Epidemiol Biom Prevention. 2001; 10(10): 1055-62.

9. Tokui N, Yoshimura T, Fujino $Y$, Mizane T, Hoshiyama $Y$, Yatsuya $H$, et al. Jacc Study Group. Dietary habits and stomach cancer risk in the Jacc study. J Epidemiol. 2005; (Suppl.2):S98-108.

10. Libonati VF, Andrade JC, Guerreiro PMS. Alguns aspectos da produção de alimentos no Estado do Pará. In: Anais do Simpósio Brasileiro de Alimentação, 5 - Maceió. Faculdade de Ciências Agrárias do Pará; 1978.

11. Empresa Brasileira de Pesquisa Agropecuária. O extrativismo animal na Amazônia: o caso de uma economia ilegal. Centro de Pesquisa Agro-florestal da Amazônia. Belém; 1992.

12. Instituto Brasileiro de Geografia e Estatística. Anuário Estatístico do Brasil. Rio de Janeiro; 1966.

13. Wisniewski A, Libonati VF. Alguns aspectos de alimentação na Amazônia. Instituto de Pesquisas e Experimentação Agropecuária do Norte. Belém; 1967.

14. Wetterberg GF, Ferreira M, Brito WLS. Fauna amazônica preferida como alimento. Projeto de Desenvolvimento e Pesquisa Florestal. Brasília: PNUD; 1976.

15. Instituto Brasileiro de Geografia e Estatística. Anuário Estatístico do Brasil; 1978.

16. Comissão Estadual de Planejamento Agrícola. Diagnóstico do setor agrícola do Pará. O meio físico: características e potencialidades dos recursos naturais. Belém; 1978.

17. Bastos ML, Ferreira MA. Dificuldades e incerteza da toxicologia perante os constituintes alimentares. Soc Bras Ciênc Tecnol Alim. 1977; 23:125-40.

18. Moran J. O problema alimentar no Brasil. A importância do equilíbrio tecnológico. Soc Bras Ciênc Tecnol Alim. 1977; 23:141-50.

19. Ayres A. Alimentação na Amazônia. In: Aspecto econômico da alimentação na Amazônia. Inst Antropol Etnol Pará. 1991; 4:17-26.

20. Instituto Brasileiro de Geografia e Estatística. Pesquisa de Orçamento Familiar. 1995/96. Rio de Janeiro; 1998.

21. Instituto Brasileiro de Geografia e Estatística. Pesquisa de Orçamento Familiar. 1987/88. Rio de Janeiro; 1991.

22. Patel M, Mendall MA, Khulusi S, Northfield TC, Stratchan DP. Helicobacter pylori infection in childhood: risk factor and effect on growth. $\mathrm{Br}$ Med J. 1994; 309(6962):1119-23.

23. Goodman KJ. Implications of Helicobacter pylori infection for stomach cancer prevention. Cad Saúde Pública. 1997; 13(Suppl 1):15-25. 
24. Boffetta P. Infection with Helicobacter pylori and parasites, social class and cancer. IARC Scientific Publication. 1997; 138:325-9.

25. Kivi M, Tindberg Y. Helicobacter pylori occurrence and transmission: a family affair? Scand J Infect Dis. 2006; 38(6):407-17.

26. Ozen A, Erten D, Pehlivanoglu E. Natural history and symptomatology of Helicobacter pylori in childhood and factors determining the epidemiology of infection. J Ped Gastroenterol Nutr. 2006; 42(4):398-404.

27. Hirohata T, Kono S. Diet/nutrition and stomach cancer in Japan. Int J Cancer. 1997; (Suppl 10): 34-6.
28. Willet MC. Diet, nutrition and avoidable cancer. Env Health Persp. 1995; 103(Suppl 8):S165-70.

29. World Health Organization. Diet, nutrition and the prevention of chronic diseases. Ginebra; 2003. WHO Techn Reports, 916.

30. Hwang H, Dwyer J, Russell M. Diet, Helicobacter pylori infection, food preservation and gastric cancer risk: are there new roles for preventive factors? Nutr Rev. 1994; 52(3):75-83.

Recebido em: 28/3/2005

Versão final reapresentada em: 28/11/2005 Aprovado em: 21/2/2006 
